\title{
Understanding CSV: Ein neues Narrativ des Kapitalismus? \\ Zur Geschichte der scheinbaren Emanzipation vom neoliberalen Para- digma Milton Friedmans
}

\author{
MARC C. HÜBSCHER
}

Der CSV von Porter und Kramer wird in Theorie und Praxis intensiv diskutiert. Am Beispiel der in der Financial Times geführten Diskussion zeigt der Beitrag die sehr unterschiedlichen Auffassungen zum CSV auf. Im zweiten Teil des Beitrags wird der theoretische Kern des CSV auf ein Missverständnis der Position von Milton Friedman zurückgeführt. Abschließend wird der CSV als Narrativ gedeutet, das nicht in der Lage ist, den Kapitalismus zu relegitimieren.

Schlagwörter: Shared Value, Corporate Social Responsibility, Separationsthese, Kapitalismus

\section{Understanding CSV: A New Narrative to Reshape Capitalism?}

In theory and practice we observe an intensive discussion of the CSV. Based on the contributions regarding the CSV in the Financial Times we summarize different views on the CSV. In the second part of the paper we argue that the theoretical core argument of the CSV is based on a misunderstanding of Milton Friedman. Finally, we describe CSV as a narrative which is not able to relegitimize capitalism.

Keywords: Shared Value, Corporate Social Responsibility, Separation Thesis, Capitalism

\section{Einleitung}

Der Aufstieg des CSV-Konzepts von Porter und Kramer (2006; 2011) hat etwas Interessantes und zugleich Befremdliches an sich. Interessant doch allein deswegen, weil Porter und Kramer mit den genannten Publikationen im Harvard Business Review (HBR) offensichtlich ein relevantes Thema adressatengerecht vorstellen. Befremdlich ist es, weil bei genauerem Hinsehen nicht ganz klar wird, was das Neue am CSV ausmachen soll. Wenn aber die zweite Beobachtung einschlägig ist, dann drängt sich förmlich die Frage auf, ob Porter und Kramer überhaupt in der Lage sind, das einzulösen, was sie mit dieser „,big idea“ ankündigen: Sie sehen im CSV „our best chance to legitimize business again“" (Porter/Kramer 2011: 4). Eine Intention, die die deutschsprachige Ausgabe (Harvard Business Manager) des HBR dazu veranlasste, den Beitrag in einer Ausgabe zu veröffentlichen, die den Titel „Wirtschaft neu denken. Wie der Kapitalismus sich wandeln muss" trug und den Aufsatz "Creating Shared Value" (Porter/Kramer 2011) mit „Die Neuerfindung des Kapitalismus“ (Porter/Kramer 2012a)

\footnotetext{
Dr. Marc C. Hübscher, Tarpenbekstraße 135, D-20251 Hamburg, Tel.: +49-(0)40-46776144, Fax: +49-(0)40-320804943, E-Mail: Marc_Huebscher@web.de, Forschungsschwerpunkte: Wirtschafts- und Unternehmensethik, Organisationstheorie, strategisches Management.
} 
übersetzte. Eine sehr zugespitzte Formulierung, die aber aus meiner Sicht die Intention von Porter und Kramer sehr gut beschreibt. Porter selbst sieht in CSV die Möglichkeit, „to make a substantial and positive impact on virtually every societal need if we can open up our thinking of what capitalism really is" (Driver 2013: 423, Hervorh. d. Verf.).

Ein Blick in den operativen Kern des CSV lässt nicht wirklich Neues entdecken. Porter und Kramer (2006) binden den CSV über eine inside-out Perspektive in das portersche Wertkettenmodell (vgl. Porter 2008) sowie über eine outside-in Perspektive in das „Diamanten-Modell“ der nationalen Wettbewerbsfähigkeit (vgl. Porter 1990) ein und formulieren mit einem neuen Verständnis von Produkten und Märkten, einer neuen Sicht auf die Produktivität der Wertschöpfungskette und dem Aufbau lokaler Cluster drei Wege zur Generierung von Shared Value (vgl. Porter/Kramer 2011; Porter/Kramer 2012a; Porter/Kramer 2012b). Mit einem sehr sachlichen Blick auf die Architektur des CSV lässt sich tatsächlich kaum erklären, warum diese Idee einerseits sehr affirmativ und andererseits sehr kritisch aufgenommen wurde.

Gewiss, die Publikation einer Idee im HBR kann bereits darauf vertrauen, dass eine gewisse Wahrnehmung des Beitrags in Praxis und Theorie entsteht. Darüber hinaus kommt in diesem Fall noch der sogenannte „Porter-Effekt" (vgl. Crane et al. 2014a: 133) hinzu. Ein weiterer, aus meiner Sicht wesentlicher Punkt ist die ,semantische Aufrüstung' des CSV, mit der Porter und Kramer (2011) behaupten, dass mit einem auf dem CSV basierenden strategischen Denkrahmen eine neue Stufe des Kapitalismus erreicht werden könne (vgl. Driver 2012: 423).

Ich möchte im Folgenden das Für und Wider des CSV-Konzepts am Beispiel der Diskussion des CSV in der Financial Times darstellen. Es lässt sich dort meines Erachtens sehr gut veranschaulichen, wie die Rhetorik des CSV (vgl. Ulrich 2012: 35) mit Bezug auf damit verbundene Chancen und Risiken in der Interpretation wirkt (Kap. 2). Diese unterschiedliche Wahrnehmung des CSV sagt aber noch nichts über den eigentlichen Kern aus, den Porter und Kramer gewissermaßen als grundlegende Annahme in den strategischen Denkrahmen einführen. Was macht diesen Denkrahmen aus? Meines Erachtens lässt sich diese grundlegende Annahme der simultanen Berücksichtigung von ökonomischen und gesellschaftlichen Zielen nur verstehen, wenn sie in den Kontext der friedmanschen Doktrin der ökonomischen Verantwortung des Unternehmens (vgl. Friedman 1970) gestellt wird (Kap. 3). Abschließend werde ich CSV als weiterhin naives Narrativ einer Re-Legitimierung des Kapitalismus bezeichnen (Kap. 4).

\section{Rezeption des CSV am Beispiel der Financial Times}

Porter und Kramer (2011) stellen einen neuen strategischen Denkrahmen vor, den sie als Creating Shared Value bezeichnen. Ich habe an anderer Stelle (vgl. Hübscher 2015) zu zeigen versucht, dass sich das Ergebnis CSV als ein längerer Entwicklungsprozess von Porter und Kramer in Bezug auf die Vereinbarkeit von ökonomischen und gesellschaftlichen Zielen bzw. genauer die Integration von gesellschaftlichen Zielen in die ökonomische Zielfunktion von Unternehmen verstehen lässt. Aber erst in ihrem letzten Artikel führen Porter und Kramer (2011) CSV als Konzept - als neuen strategischen Denkrahmen - explizit ein und bieten es als Lösung an, um den Kapitalismus 
als zunehmend stärker kritisierte Wirtschaftsform (vgl. etwa aktuell Sedlacek/Graeber 2015; Streeck 2015) wieder - gewissermaßen affirmativ - in die Gesellschaft zurückzuführen.

An der Einschätzung der Möglichkeiten allerdings, die Porter und Kramer mit dem CSV verbinden, scheiden sich die Geister, wie wir es den Kommentaren zum CSV in der Financial Times (FT) entnehmen können. Beginnen möchte ich mit der fundamentalen Kritik von Crane und der nicht überraschenden affirmativen Gegenposition von Kramer. Darüber hinaus finden sich Kommentare, die weniger klar zuzuordnen sind. Während Hahn den CSV als kommunikationsstrategisch wichtige Entwicklung (bei allen konzeptionellen Schwierigkeiten) für die Einbettung des CSR-Diskurses in die management school agenda beschreibt, steht Donaldson näher zur Kritik von Crane, da die Simplifizierungen des CSV sehr grundsätzliche Herausforderungen im Managementdiskurs verhindern. Lütge und von Liel hingegen scheinen im CSV eine theoretische Offenbarung für die Verbindung von gesellschaftlichen und ökonomischen Zielen zu sehen, die kritiklos sogar über das Anliegen von Porter und Kramer hinaus gedeutet werden kann. Der Beitrag von Dyllick scheint mir insgesamt ein gutes Beispiel zu sein, um die Wirkung des ,süßen Gifts', dass das Narrativ CSV versprüht, zu veranschaulichen. Zunächst schien er überzeugt, dass der CSV eine gute theoretische Weiterentwicklung in Richtung Nachhaltigkeit ist, während er es in dem Beitrag der Zeit zu überlassen scheint, ob der CSV ein hilfreiches oder eher verhinderndes Denkgebäude ist, um gesellschaftliche Anliegen in den Managementdiskurs ernsthaft zu integrieren.

Crane (2014), der mit seinen Koautoren eine sehr fundierte und detaillierte Kritik am CSV (vgl. Crane et al. 2014a) vorgetragen hat, kritisiert in der FT: „CSV risks misleading today's business student about the reality of the societal challenges facing business“. Im Kern sieht er (vgl. Crane 2014) drei wesentliche Kritikpunkte am CSV (vgl. auch Beschorner 2013; Crane et al. 2014a): Erstens wird die Annahme von Porter und Kramer, dass viele (nicht alle, vgl. Porter/Kramer 2006: 92; 2011: 17) ökonomische und gesellschaftliche Ziele im positiven Sinne in einen Ausgleich (win-win) überführt werden können, nicht geteilt und vielmehr davon ausgegangen, dass im Gegenteil viele gesellschaftliche Anliegen konfliktär zu rein ökonomischen Zielen stehen (vgl. Scholz/de los Reyes 2015; sowie in dieser Ausgabe). Zweitens kritisiert Crane (2014), dass die „compliance with legal and ethical norms" allzu naiv von Porter und Kramer behauptet wird, ohne gebührend zu berücksichtigen, dass genau diese Annahme von Unternehmen nicht eingehalten wurde und u.a. dieses zu einer (neuen) „Legitimationskrise des Spätkapitalismus" (Habermas 1973) beiträgt (vgl. Crane 2014: 2; Crane et al. 2014b: 152). Schließlich kritisiert Crane (2014), dass Porter und Kramer im Kern lediglich vorschlagen, dass die Krise des Kapitalismus aus der Stärkung der ökonomischen Verantwortung der Unternehmen mit einem Fokus auf „doing good“ (Crane 2014) erreicht werden könne. Die gewissermaßen Transformation von gesellschaftlichen Anliegen in business cases ist aber nichts Neues und im strategischen CSR bereits seit Längerem bekannt (vgl. etwa Schreck 2012).

Wenn sich aber Executives unter dem legitimitätserzeugenden Schirm des CSV von Porter und Kramer versammeln, dann müssen weiterhin viele gesellschaftliche Probleme unter dem friedmanschen Diktum der ökonomischen Verantwortung des Unternehmens an andere, jedenfalls nicht an erwerbswirtschaftliche Unternehmen, adres- 
siert werden. Die Idee, dass „(b)usinesses have to learn to work together not against each other in solving social problems" (Crane 2014: 2) kommt damit nicht nur nicht recht in den Blick, sondern wird in erster Linie negiert bzw. angepasst, so dass ein ,work together' nur in eben den Fällen geboten ist, wo die gesellschaftlichen Anliegen in ein Geschäftsmodell überführt werden können.

Dem gegenüber hat Kramer (2014) in der FT klargestellt, CSV sei eine der „,most promising avenues of competitive advantage and wealth creation in the world today". Diese Sicht ist nur dann zu verstehen, wenn das Verständnis von Porter und Kramer in Bezug auf die bestehenden CSR-Praxen bekannt ist (vgl. Hübscher 2015). Sie reduzieren CSR entweder auf ein „pure giving“ (Driver 2012: 424) oder aber auf ein „on what not to do" (Kramer 2014: 1, Hervorh. d. Verf.). Mit diesem Verständnis von CSR, dem sie am Ende den CSV substitutiv entgegenstellen (vgl. Porter/Kramer 2011: 16) verbinden sie das Programm, gesellschaftliche Anliegen „into the mainstream of management" (Driver 2012: 427) zu bringen und es nicht weiter in einer „side agenda“ (ebd.), wie sie CSR und Philanthropie bezeichnen, zu führen.

\begin{abstract}
„Business schools can continue to treat social issues as mere peripheral constraints on the core skills of business, but this blind spot will yield graduates that lack the knowledge to recognise immense opportunities and the skills to meet their future employers' needs. Or, business schools can develop courses in social impact (...) Those courses will look nothing like the current offerings in CSR and ethics; they will teach knowledge and skills, not constraints and admonitions" (Kramer 2014: 2, Hervorh. d. Verf.).
\end{abstract}

Im Übrigen scheinen sie die Richtigkeit des CSV über die positive Resonanz in der Praxis zu rechtfertigen und wähnen sich daher auf dem richtigen Weg (vgl. Porter/Kramer 2014: 149; Kramer 2014).

Hahn (2014) und Donaldson (2014) sehen in gedanklicher Nachbarschaft zu Crane (2014) auch sehr klar die engen Grenzen der Berücksichtigung gesellschaftlicher Anliegen im erwerbswirtschaftlichen Geschäftsgebaren sensu Porter und Kramer. Während Hahn (2014) zwar der Überzeugung ist, dass die Publikationen zum CSV für alle diejenigen, die CSR und Nachhaltigkeit unterrichten und betreiben, „a gift from heaven" (ebd.) ist, wenn ein angesehener ,business guy ${ }^{61}$ wie Porter in einem ebenfalls in Theorie und Praxis angesehenen Publikationsorgan affirmativ über die Vereinbarkeit von gesellschaftlichen und ökonomischen Anliegen schreibt. Gleichwohl sieht er doch auch deutlich die Grenzen des mit dem CSV verbundenen mental models (vgl. dazu Hartman/Werhane 2013). Die simple Logik des CSV inkorporiert nämlich eine „easy option“ (Hahn 2014: 1), die da heißt: entweder führen gesellschaftliche Anliegen in ein business model (win-win) oder aber sie sind zu ignorieren (vgl. ebd). An genau diesem Punkt setzt seine Kritik an. Porter und Kramer würden mit der Logik des CSV einfache Lösungsmuster für oftmals komplexe Probleme anbieten, die aber dann zu unterkomplexen Lösungsschritten führen können. Dem entgegen müssten Management und Managementnachwuchs vielmehr darin geübt werden, gesellschaftliche

So Porter über sich selbst: „I am not a social problem guy, I am a business guy, who helps business make money" (Quelle: https://www.youtube.com/watch?v=0ilh5YYDR2o (zuletzt abgerufen am 20.04.2015). 
Herausforderungen mit einem ,thinking out of the box ${ }^{`}$ anzugehen. Hahn (2014) sieht genau darin aber einen Gegenentwurf zum CSV, weil Simplizitität durch „paradoxical thinking“ (ebd.) ersetzt, Komplexität gewissermaßen bejaht werden müsste (vgl. Kirsch et al. 2009: 168ff.), und damit Inkompatibilitäten zwischen gesellschaftlichen und ökonomischen Zielen zu einem Startpunkt einer Diskussion über bestehenden Geschäftsroutinen und -lösungen führen können. „But if we want future business leaders to make a meaningful contribution to sustainability, we also need to train them to think beyond profit making and business success as defined by standard finance and strategy to come up with more radical solutions in the first place" (Hahn 2013: 3). Donaldson (2014) wird an dieser Stelle noch deutlicher, weil er gewissermaßen die (in der Wissenschaft nicht unbekannte) Polylingualität von ökonomischen Organisationen (vgl. Wieland 2005; Hübscher 2011) ausdrücklich anerkennt und damit hervorhebt, dass ein Projekt CSV kaum in der Lage sein kann shared value zu schaffen, wenn es die polylinguale Struktur gesellschaftlicher Anliegen wieder in die monolinguale Struktur einer rein ökonomischen Verantwortung einhegt (vgl. Wieland/Heck 2013: 15ff.): „Shared Values that are lost in transaction“ (Donaldson 2014). Shared Value kann nur geschaffen werden, wenn die gesellschaftlichen und ökonomischen Ziele (notwendig) simultan und (hinreichend) effizient bearbeitet werden können. Ich denke, es ist sehr hilfreich, wenn Donaldson (2014) in diesem Zusammenhang eine Hammer-Nagel-Metapher bedient. ${ }^{2}$ Denn die Logik des Hammers erfordert, Probleme wie einen Nagel aussehen zu lassen. Ehedem die Herausforderungen gesellschaftlicher Anliegen verstanden werden, erhalten sie so - gewissermaßen „problemvergewaltigend“ (Kirsch et al. 2009: 168ff.) - den Mantel eines Nagels.

Auch hier wird, wie bereits bei Hahn (2014), die monolinguale Logik des CSV in Bezug auf die ökonomistische Verantwortung des Unternehmens kritisiert. Anders aber als bei Hahn (2014), der aus einem ,Managementdenken' heraus eine Erweiterung fordert, die nicht im CSV angelegt ist, sieht Donaldson sehr grundsätzlich das Problem, dass moralische Probleme nicht in einem „optimisation“-Sprachspiel verhandelt und gelöst werden können, sondern in einem „,commitment“-Sprachspiel, das weniger auf einer konsequentialistischen, sondern vielmehr auf einer deontischen Logik beruhe (vgl. Donaldson 2014). CSV richtig umgesetzt bedarf immer einer Antwort auf die Frage, was the nature of the business ist. Donaldson definiert business als „a form of cooperative activity for the purpose of efficiently achieving mutual and collective value" (ebd.) und um diese Definition mit Leben zu füllen, bedarf es mehr als einer effizienzorientierten Sicht der ökonomischen Verantwortung des Unternehmens.

Lütge und von Liel (2014, sowie in dieser Ausgabe) hingegen kritisieren die sehr kritische Sicht auf CSV und betonen das Potenzial, mit dem Porter und Kramer die Diskussion in Richtung einer „more advanced social responsibility of business" (ebd.) bewegen. Der positive Kern des CSV wird von Lütge und von Liel (2014) in dem vorgenommenen Perspektivenwechsel gesehen. „It changes the perspective from one where corporations harm society and must therefore give back to society, to one where corporations are becoming part of the solution to social and societal challeng-

2 "If the only thing one has is a hammer, then everything starts to look like a nail" (Donaldson 2014). 
es“ (ebd.). Gleichwohl kann dieser Perspektivenwechsel nicht dazu führen, dass alle gesellschaftlichen Probleme durch Unternehmen gelöst werden können (vgl. Porter/Kramer 2011: 17), aber das schmälert aus Sicht von Lütge und von Liel (2014) nicht die „fundamental benefits of the CSV concept“. Allein die Empfehlung an Unternehmen, systematisch nach win-win Situationen in der simultanen Berücksichtigung von gesellschaftlichen und ökonomischen Werten zu suchen, sei ein wesentlicher Schritt für ein modernes Management. Erst dann nämlich, wenn diese professionelle Suche nicht erfolgreich, bzw. nicht erfolgsversprechend ist, müssten Unternehmen „with trade-offs of business and other interests“ (ebd.) umgehen. Inwieweit dieser weitere Umgang mit gesellschaftlichen Anliegen, die nicht in einer win-win Situation abbildbar sind, von Porter und Kramer ebenfalls als Unternehmensaufgabe, wie Lütge und von Liel (2014) suggerieren, verstanden wird, ist meines Erachtens spekulativ und wird von Porter und Kramer selbst sehr unklar beschrieben. Einerseits erklären sie, dass CSV ein Denkrahmen ,about solving societal problems in order to create economic value“ (Porter/Kramer 2014: 149) sei und andererseits ergänzen sie, „that legal compliance and a narrow sense of social responsibility are prerequisites to creating shared value“ (Porter/Kramer 2014: 150). Wie dieser „,narrow sense of social responsibility“ zu verstehen und auszugestalten ist, bleibt offen (vgl. Crane et al. 2014b: 152). Dieses nicht unwesentliche Problem bekommen Lütge und von Liel nicht recht in den Blick, sehen sie doch schon den fundamentalen Schritt des CSV in der Anpassung des mental models des Managements in Praxis und Ausbildung „to look first for CSV opportunities - meaning: win-win solutions - before turning to solve the remaining trade-offs" (Lütge/von Liel 2014). Um es klar zu sagen: Ich sehe in der Formulierung von Lütge und von Liel eine erweiternde Interpretation des CSV. Während die systematische Identifikation von „Corporate Social Opportunities“ (Grayon/Hodges 2004; vgl. auch Porter/Kramer 2006: 92) den Kern des CSV bezeichnet, ist der weitere Aspekt „to solve the remaining trade-offs“ eine Interpretation, die aus meiner Sicht nur mit viel Wohlwollen in der Argumentation von Porter und Kramer gelesen werden kann. Ich denke, Porter und Kramer würden derartiges selbst nicht formulieren.

Dyllick (2014) bezieht einen Standpunkt zum CSV ein, der nicht nur Für und Wider des CSV ernst nimmt, sondern zuallererst das , süße Gift des CSV kenntlich macht, das durch Porter und Kramer in Managementtheorie und -praxis versprüht wird. In Kenntnis der Artikel von Porter und Kramer sah Dyllick (2014) nämlich zunächst einen wesentlichen Schritt, oder gar einen ,Durchbruch “, in redirecting the debate on the purpose of business“. Die Attraktivität des CSV liege aus Dyllicks Sicht in der programmatischen Intention von Porter und Kramer, ,that the purpose of the corporation had to be redefined as creating shared value - creating economic values in a way that also creates value for society by addressing its challenges and needs" (ebd.). In dieser Wahrnehmung sieht Dyllick ein Versprechen „to connect business and society in a more integrated and holistic way" (ebd.). Diese affirmative Aufnahme des CSV wurde aber getrübt durch die Kenntnisnahme der Kritik am CSV durch Crane et al. (2014a). Die brillante Rhetorik von Porter und Kramer als ,süßes Gift' schien also zunächst bei Dyllick umfänglich zu wirken. Nach Abwägung beider Argumente kommt Dyllick zu einem bemerkenswerten Schluss: Einerseits - in der Diskussion mit der Managementpraxis - ist der CSV ein erfolgreiches Vehikel zur Berücksichtigung von gesellschaftlichen Anliegen im business case, ,because we know this relates to the 
world that they live in“ (ebd.), und andererseits - in der wissenschaftlichen Diskussion - „we freely discuss the limitations and shortcomings of such win-win approaches" (ebd.). Dyllick dürfte sehr genau wissen (vgl. Dyllick 1989), dass bereits etwa Drucker (2002a/1974: 78) ,gesellschaftliche Probleme als wirtschaftliche Chance“ und Innovationspool für Unternehmen verstanden hat, so dass ohne große Schwierigkeiten angenommen werden kann, dass gesellschaftliche Bedürfnisse die Basis unternehmerischen Handelns sind, und erst dann, wenn gesellschaftliche Bedürfnisse in Bedarf gewandelt werden, ein business case entsteht (vgl. ebd.: 78f.).

Die Rekapitulation der Diskussion des CSV in der FT zeigt aus meiner Sicht, dass insbesondere aufgrund der intensiven Rezeption des CSV in der Praxis eine gründliche Würdigung des CSV in Bezug auf das, was er leisten kann und wo seine Grenzen sind, erforderlich ist. Neben dem nicht überraschend befürwortenden Beitrag von Kramer (2014), stehen mit Crane (2014), Donaldson (2014) und Hahn (2014) sehr ernstzunehmende Kritiken im Raum. Dyllick (2014) sieht dagegen in der Doppeldeutigkeit der Wahrnehmung des CSV in Managementtheorie und -praxis eine Chance für die ernsthafte Berücksichtigung von gesellschaftlichen Anliegen im Geschäftsmodell und konkludiert, dass ,we realize that they both have their own truth". Lütge und von Liel (2014), ganz im gedanklichen Verbund mit Porter und Kramer, empfehlen den CSV als wichtigen Bestandteil in der Ausbildung von business schools, weil ,(i)f you are not taught to look systematically for win-win opportunities, vou will only see trade-offs".

Unter Berücksichtigung dieser Diskussion stellt sich die Frage, was eigentlich falsch läuft in der Aus- und Weiterbildung des Managements? Einerseits wird der CSV als ,neues Konzept' so intensiv und vor allem affirmativ diskutiert und andererseits kann dargelegt werden kann, dass die inhaltliche Position des CSV nicht nur seit Jahrzehnten bekannt ist, sondern auch in der CSR-Diskussion gesellschaftliche Verantwortung als business case breit diskutiert wird (vgl. Schreck 2012). Aus Sicht der Institutionalisierung der Managementausbildung ist es sehr ernst zu nehmen, dass Professoren an business schools, wie etwa Porter, CSR als side agenda zum mainstream of management wahrnehmen und so aller Wahrscheinlichkeit nach auch in ihren Programmen unterrichten. Insoweit dürfte es dann auch nicht verwundern, dass die Managementpraxis selbst, unterstellend, dass sie durch derartige Ausbildungsprogramme gelaufen ist, ein ähnliches argumentatives ,Unwohlsein' mit CSR hat wie Porter und Kramer. Aber woraus resultiert eigentlich diese Dichotomie zwischen gesellschaftlichen und ökonomischen Zielen, deren Überwindung Porter und Kramer mit dem CSV zu erreichen gedenken?

\section{Porter, Kramer und Friedman}

Ich bin davon überzeugt, dass diese Frage nicht durch die Diskussion des CSV als Endprodukt der Überlegungen von Porter und Kramer beantwortet werden kann, sondern vielmehr durch die Entwicklung hin zum CSV selbst (vgl. Hübscher 2015).

Porter entwickelte seine Ideen zum strategischen Management in den ausgehenden 1980er Jahren an der Harvard University, einer Institution, die bereits zuvor eine Tradition der Forschung zum strategischen Management aufwies. Learned, Christensen, Andrews und Guth haben etwa in den 1960er und 1970er Jahren umfangreiche Publikationen zum strategischen Management vorgelegt. Learned et al. (1967: 491) waren überzeugt, dass die strategische Dimension eines Unternehmens vier „principal as- 
pects“ der Strategieformulierung umfasst: „(1) appraisal of present and foreseeable opportunity and risk in the company's environment, (2) assessment of the firm's unique combination of present and potential corporate resources and competences, (3) determination of the noneconomic personal and organizational preferences to be satisfied, (4) identification and acceptance of the social responsibility of the firm". Porter (1999: 24) verweist unter der Überschrift „Rückblick: Der klassische Ansatz zur Formulierung von Strategien" in seinem in den 1980er Jahren erschienenen Werk „Wettbewerbsstrategie“ auf diese Historie. Aber bereits in der Einleitung grenzt Porter (1999: 27) die ,identification and acceptance of the social reponsibility of the firm" (Learned et al. 1967: 491) auf den „Einfluß gesellschaftlicher Erwartungen“ ein. AuBerdem kann ich nur vermuten, dass die „noneconomic personal and organizational preferences to be satisfied“" (Learned et al. 1967:491), die - nicht mehr explizit thematisiert - auf eine ökonomische Dimension reduziert werden, wenn Porter (1999: 70) „Wettbewerbsstrategien als die Wahl offensiver oder defensiver Maßnahmen, um (...) einen höheren Ertrag auf das investierte Kapital zu erzielen“ versteht. Mit diesem ersten Schritt vollzog Porter eine Entwicklung im strategischen Management, die Freeman et al. (2010: 91) als ,the effect of reinforcing the economic theory upon which much of the field of strategic management was already based" beschreiben. Ich denke, dieser Schritt der erfolgreichen (Re-) Ökonomisierung des strategischen Managements durch Porter ist wichtig, um die gedankliche Entwicklung des CSV zu verstehen.

Unter dem Einfluss des industrieökonomischen Denkens war es für Porter selbstverständlich, Strategie und Wettbewerbsvorteile in einem „optimisation“-Sprachspiel (Donaldson 2014) zu entwickeln, das sich sehr gut in das neoliberale Konzept von politischer und ökonomischer Freiheit eines Milton Friedmans (2010) einhegt. Nicht zufällig ist daher auch meines Erachtens die Abarbeitung an der neoliberalen Position von Milton Friedman (1970) sehr zentral für die Entwicklung des CSV.

In der Rechtfertigung des CSV argumentieren Porter und Kramer: „(w)e ground the opportunity for CSV in new learning about the effect of externalities on the firm, including our own work on the environment, urban poverty, global health, and other social issues over the past 20 years" (Porter/Kramer 2014: 149). Tatsächlich aber lässt sich neben dieser Motivation der eigentliche Kern des CSV auf die Beschäftigung von Porter und Kramer mit philanthropischen Engagement in den vergangenen rund 15 Jahren zurückführen (vgl. Hübscher 2015).

In Porter und Kramer (1999) - ganz in der Tradition von Friedman (1970) - entwickeln sie Vorschläge, wie philanthropisches Engagement auf Gesellschafter-Ebene mit Instrumenten des strategischen Managements effizienter gestaltet werden könne. Hier argumentieren Porter und Kramer nicht wie philanthropisches Engagement auf Gesellschafts-Ebene gerechtfertigt und eingesetzt werden kann, weil sie ganz offensichtlich Friedman (2010: 167) folgen: „(w)enn die Aktiengesellschaft Zuwendungen macht, hindert sie den einzelnen Aktionär daran, selbst zu entscheiden, wie er seine Mittel verwenden könne“. ${ }^{3}$ Diese eigene Entscheidung der Mittelverwendung des einzelnen dass es effizient und damit empfehlenswert ist, wenn Anteilseigner charities über das Unterneh- 
Gesellschafters versuchen Porter und Kramer zu professionalisieren. In dieser Diskussion fehlen freilich jedwede Bezüge zu CSR, weil ganz offensichtlich die Verantwortung des Unternehmens eine ökonomische ist, die für die Mittelerwirtschaftung zu sorgen hat (vgl. Friedman 1970).

Die eigentliche ,Geburtsstunde' des CSV, auch wenn er so in dem Zeitpunkt nicht explizit eingeführt wird, ist meines Erachtens die ökonomische Argumentation von Porter und Kramer für ein philanthropisches Engagement auf Gesellschafts-Ebene, denn: „they give up tremendous opportunities to create greater value for society and themselves. Context-focus philanthropy does not just address a company's selfinterest, it benefits many through broad social change" (Porter/Kramer 2002: 14). Auch damals, als von einer höheren Evolutionsstufe des Kapitalismus (vgl. Driver 2012: 423) noch nicht die Rede war, endeten Porter und Kramer (2002: 12) mit der Überzeugung, dass Corporate Philanthropy „a vastly more powerful way to make the world a better place" darstelle.

Dieser Schritt aber, der das philanthropische Engagement von der Gesellschafter-auf die Gesellschafts-Ebene hebt, ist für Porter und Kramer (2002: 2) in Abgrenzung zu Friedman (1970) begründungsbedürftig. Sie identifizieren zwei implizite Annahmen, die aus ihrer Sicht Friedman (1970) veranlassten, philanthropisches Handeln auf GesellschaftsEbene abzulehnen:

„The first is that social and economic objectives are separate and distinct, so that a corporation's social spending comes at the expense of its economic results. The second is the assumption that corporations, when they address social objectives, provide no greater benefit than is provided by individual donors" (Porter/Kramer 2002: 2).

Allerdings geben Porter und Kramer (ebd.) Friedman Recht, sofern Unternehmen philanthropisches Engagement unstrukturiert und -fokussiert betreiben. In dem Moment aber, in dem Unternehmen erkennen, dass sie durch philanthropisches Engagement den Wettbewerbskontext (competitive context) strukturiert verbessern können, um „a company's long-term business prospects“ (ebd.) zu erhöhen, kann die erste Annahme widerlegt werden. Darüber hinaus kann die Nutzung von Unternehmenskompetenzen zur Verbesserung des Wettbewerbskontexts zu einem Leverage-Effekt „in support of charitable causes“ (ebd.) im Vergleich zur Gesellschafter-Ebene führen, das aus ihrer Sicht auch die zweite Annahme widerlegt.

Es ist spekulativ, ob die Dichotomie von gesellschaftlichen und ökonomischen Zielen bereits bei Friedman selbst in dieser Deutlichkeit angelegt war, oder aber erst in der Interpretation von Friedman durch Porter und Kramer und vielen anderen Ökonomen. Ich kann mir aber kaum vorstellen, dass Friedman eine Investition in die Verbesserung der „long-term business prospects“ abgelehnt hätte, wenn sie durch das Unternehmen strategisch gut begründet werden kann. Ich denke, man kann sogar so weit gehen zu sagen, dass wenn die Verbesserung des Wettbewerbskontexts dazu

men verteilen. Diese Möglichkeit ist aus seiner Sicht aber ein Ärgernis, weil es persönliche Anliegen aus Effizienzgründen, nämlich der steuerlichen Abzugsfähigkeit, auf die GesellschaftsEbene hievt. Hier ist Friedman (2010: 167) konsequent und fordert daher die Abschaffung der Körperschaftsteuer (vgl. auch Friedman 2010: 163f.). 
führt, dass die Erfolgsaussichten des jeweiligen Unternehmens nachhaltig verbessert werden, dann ist die Durchführung dieser Maßnahmen durch das Paradigma der ökonomischen Verantwortung des Unternehmens, wie Friedman es vertrat, nicht nur abgedeckt, sondern durchaus geboten. Eine Strategie der kurzfristigen Gewinnmaximierung darf an dieser Stelle nicht mit dem Statement verwechselt werden, das „die verfügbaren Mittel möglichst Gewinn bringend eingesetzt und Unternehmungen unter dem Gesichtspunkt der größtmöglichen Profitabilität geführt werden müssen“ (Friedman 2010: 164).

Auf die weitere Entwicklung des CSV durch Porter und Kramer (2006; 2011) möchte ich hier nicht vertieft (vgl. dazu Hübscher 2015), lediglich kursorisch eingehen, auch wenn klar sein sollte, dass die aktuelle Diskussion des CSV mehrheitlich von diesen Beiträgen handelt (vgl. Kap. 2). In meiner Wahrnehmung wird nämlich die Idee des CSV, wie sie bereits von Porter und Kramer 2002 eingeführt wurde, durch ihren Artikel von 2006 in einen CSR-Kontext dergestalt eingebunden, dass sie den CSV (dann in Form von neuen Geschäftsmodellen auf Basis von gesellschaftlichen Zielen) und Corporate Philanthropy (in Form von Verbesserungen des Wettbewerbskontextes) als strategisches CSR in Abgrenzung zu einer reaktiven CSR vorschlagen. In ihrem jüngsten Artikel (vgl. Porter/Kramer 2011) wird dann der CSV explizit, d.h. in Abgrenzung zu CSR in Stellung gebracht und insbesondere mit der normativen Behauptung aufgeladen, dass der CSV „our best chance to legitimize business again“" (ebd.: 4) sei.

An der Argumentation von Porter und Kramer für den CSV und der durch Beiträge der Autoren dokumentierten Entwicklung dorthin scheinen mir zwei Aspekte wesentlich, die bei genauerer Betrachtung sogar einander bedingen. Der eine Aspekt ist die Dichotomie von ökonomischer und gesellschaftlicher Verantwortung, die Friedman (1970; 2010) beschäftigte und die in der Argumentation von Porter und Kramer auf eine quasi einfach zu widerlegende Dichotomie von gesellschaftlichen und ökonomischen Zielen reduziert wurde. Der zweite Aspekt betrifft die Wahrnehmung von CSR durch Porter und Kramer, die von Wirtschaftsethikern als „straw man“ (Beschorner 2013: 109; Crane et al. 2014: 134) bezeichnet wurde.

Die Kernfrage ist meines Erachtens nicht, ob ökonomische und gesellschaftliche Ziele miteinander vereinbart werden können. Diese Schein-Dichotomie verkennt nämlich grundsätzlich die Innovationskompetenz von Unternehmen, die nicht immer vorhanden, aber für die Entwicklung neuer, profitabler Geschäftsmodelle erforderlich ist (vgl. Priddat/Seele 2008; Prahalad/Krishnan 2009; Hamel 2013). Bereits Drucker (2002b/1974: 57) hatte darauf hingewiesen, dass

„gesellschaftliche Ziele keine bloßen Absichtserklärungen sein können (...). Diese Ziele werden nicht benötigt, weil der Manager Verantwortung gegenüber der Gesellschaft trägt. Sie werden benötigt, weil der Manager Verantwortung gegenüber dem Unternehmen trägt".

Darüber hinaus hat Friedman (1970: 5) - jenseits einer Geschäftsmodelllogik für soziale Anliegen - bereits sehr genau gesehen, dass es durchaus im ,long-run interest of a corporation“ liegen kann, ,to devote resources to providing amenities to that community or to improving its government". All das aber begründet sich nicht in einer wie auch immer gearteten - gesellschaftlichen Verantwortung, sondern ausschließlich 
aus der umfänglichen Wahrnehmung der ökonomischen Verantwortung von Unternehmen.

Wenn Klarheit darüber hergestellt werden kann, dass es nicht um die Vereinbarkeit von gesellschaftlichen und ökonomischen Zielen geht, sondern um die Dichotomie von gesellschaftlicher und ökonomischer Verantwortung, um die es Friedman (1970) gelegen war, dann zeigt sich der Gegensatz - auch im CSV - weiter in der Separationsthese von Freeman (1994: 412), nach der ,(t)he discourse of business and the discourse of ethics can be seperated so that sentences like, ' $\mathrm{x}$ is a business decision' have no moral content, and ' $\mathrm{x}$ is a moral decision' have no business content". Verantwortung als nicht nur rechtlichen, sondern insbesondere auch moralischen Begriff, lässt sich mit Heidbrink (2007: 11) ,nicht anders denken als das Einstehen für eine begangene oder zu vollziehende Handlung, die jemanden gerechtfertigterweise zugeschrieben werden kann". Diese Zuschreibung transzendiert ökonomische Rechtfertigung. Mit dieser Beschreibung fängt die Problemdiskussion erst an, führt aber genau in den Kern, den Freeman (1994: 412; vgl. auch Freeman et al. 2010: 6ff.) als die Überwindung der Separationsthese beschrieben hat, nämlich der Kenntnisnahme, dass business nie in einem moralischen Vakuum prozessiert wird:

"There is always a context to business, and that context is moral in nature. It is only by recognizing the moral presuppositions of business theory, refining them, testing them by living differently, and revising them that we can invent and reinvent better ways to live" (Freeman 1994: 412).

Sofern aber, und das ist aus meiner Sicht der Rahmen in dem Porter und Kramer (2006; 2011) CSV explizit entwickeln, an einer „modellplatonisch“ (Albert 1998: 114) getriebenen Konzeption des Unternehmens in seiner ausschließlich ökonomischen Verantwortung - gewissermaßen im Annahmenbereich - festgehalten wird, kann freilich die Frage einer (erweiterten) gesellschaftlichen Verantwortung von Unternehmen definitionsgemäß gar nicht weiter diskutiert werden. Zudem scheint mir die von Porter und Kramer (2002) offenbar durchgeführte Emanzipation von Friedman nicht zu greifen, da Friedman nicht primär an der Unvereinbarkeit von gesellschaftlichen und ökonomischen Zielen interessiert war, sondern an der Unvereinbarkeit von gesellschaftlichen und ökonomischen Anliegen in der Verantwortung von Unternehmen. Wenn die gesellschaftliche Verantwortung von Unternehmen ausschließlich, und das ist das Credo von Friedman (1970; 2010), in der Gewinnmaximierung liegt, dann reduziert sich gesellschaftliche Verantwortung auf die ökonomische Dimension. Wie diese ökonomische Verantwortung wahrgenommen wird, ob mit oder ohne Berücksichtigung von sozialen Zielen, ist zunächst nicht von Belang. Relevant werden diese freilich, wenn die langfristige Überlebensfähigkeit und Gewinnmaximierungsabsicht des Unternehmens eingeschränkt oder bedroht wird.

Ich denke, diese Vermischung von Ziel- und Verantwortungsdimension von Unternehmen trägt auch nicht unwesentlich dazu bei, dass Porter und Kramer CSR als jenen ,straw man' zeichnen, der Wohltaten aus der Gewinnverwendung organisiert und nicht den operativen Kern der Gewinnerwirtschaftung, der erst durch den CSV in eine strategische CSR mündet (vgl. Porter/Kramer 2006). Man kann den Autoren ein gewisses Unwohlsein mit dem Begriff der CSR unterstellen, weil dieser die gesellschaftliche Verantwortung von Unternehmen thematisiert. Folglich konstruieren Porter und 
Kramer (2011) den CSV nicht mehr als Bestandteil einer CSR, sondern stellen ihn schließlich der CSR grundsätzlich entgegen.

Aus meiner Sicht lassen sich die Entwicklung und die Grenzen des CSV sehr anschaulich in einer Matrix des Umgangs mit den Dichotomien ökonomische vs. gesellschaftliche Ziele und ökonomische vs. gesellschaftliche Verantwortung darstellen (vgl. Abb. 1).

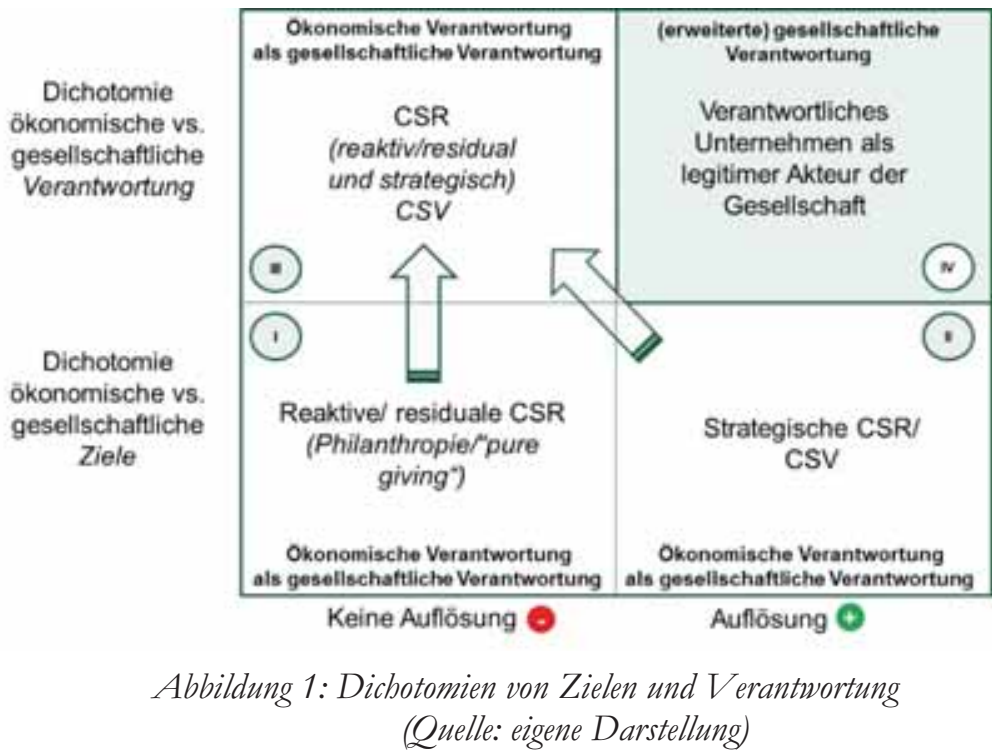

Sofern eine Dichotomie von ökonomischen und gesellschaftlichen Zielen angenommen wird, ist das Ziel des Unternehmens eine Gewinnmaximierung unter ausschließlich ökonomisch getriebenen Zielen. Wenn in dieser Situation (Quadrant I) das wenig klar definierte Phänomen CSR (vgl. Freeman et al. 2010: 235f.; Porter 2003: 1) in Unternehmen diskutiert wird, dann in Formen der reaktiven (Porter/Kramer 2007: 27) oder residualen (Freeman et al. 2010: 257) CSR, die unterstellen, „that there is a moral obligation and/or a number of good practical reasons for corporations to give back to the society some of the value they have created" (ebd.). In dem Moment, in dem die Dichotomie zwischen ökonomischen und gesellschaftlichen Zielen aufgelöst und durch eine integrative Sicht ersetzt wird (vgl. Porter/Kramer 2006), öffnet sich der Weg (Quadrant II) zu einer strategischen CSR, die es erlaubt und fordert, gesellschaftliche Ziele und Anliegen ernst zu nehmen und für die Suche nach neuen und innovativen Geschäftsmodellen zu nutzen. Beide Fälle bewegen sich aber in einer stillschweigenden Dichotomie zwischen gesellschaftlicher Verantwortung als ökonomische Verantwortung (Friedman-Perspektive) und einer (erweiterten) gesellschaftlichen Verantwortung, die das Unternehmen als „Corporate Citizenship“ ernst nimmt (vgl. Crane/Matten 2010: 76). CSR umfasst auf dieser Diskussionsebene im Fall einer bestehenden Dichotomie von gesellschaftlicher und ökonomischer Verantwortung des Unternehmens (Quadrant III) sowohl ein residuales als auch ein strategisches Vorgehen. Eine integrierte Vorstellung von CSR (vgl. Freeman et al. 2010: 258), im Sinne einer wohlverstandenen gesellschaftlichen Verantwortung von Unternehmen, kommt 
aber erst in den Blick, wenn die Dichotomie von ökonomischer vs. weiterer gesellschaftlicher Verantwortung voraussetzungsfrei aufgelöst wird (Quadrant IV).

Wenn die Überwindung der Dichotomie zwischen ökonomischer und (erweiterter) gesellschaftlicher Verantwortung als eine Aufgabe der Widerlegung der Seperationsthese sensu Freeman gelesen wird, dann wird meiner Erachtens erst jetzt der Weg frei, der erlaubt, das zu bearbeiten, was sich Porter und Kramer (2011) in Form einer ReLegitimierung auf die Agenda geschrieben haben, nämlich „how to reinvent capitalism“. Mit diesem Schritt würden sie tatsächlich die Annahmen Friedmans emanzipatorisch herausfordern und an einem neuen Narrativ im Sinne einer legitimierenden Erzählung ,about how capitalism can be a force for good in the world“ (Freeman et al. 2010: 267) arbeiten.

\section{4. $\quad$ Fazit}

In der Entwicklung des CSV haben sich Porter und Kramer intensiv an der Widerlegung der scheinbaren Dichotomie von gesellschaftlichen und ökonomischen Zielen abgearbeitet, um, nach rund 15 Jahren, zu dem Ergebnis zu kommen, dass in Zeiten einer erneuten Legitimationskrise des Kapitalismus der CSV als „best chance to legitimize business again" (Porter/Kramer 2011: 4) in Betracht kommt.

Der Versuch der beiden Autoren, über den CSV eine Re-Legitimierung des Kapitalismus zu konstruieren, lässt den CSV als neues Narrativ des Kapitalismus erscheinen (vgl. Hübscher 2015), der in der Sprache von Ökonomen und an business schools ausgebildeten Executives formuliert ist. Es ist freilich für Executives attraktiver über value creation zu sprechen, als sich mit dem sperrigen und diffus anmutenden Begriff responsibility auseinanderzusetzen. Narrative sind ,perceived sequences of non-randomly connected events" (Bottici 2010 zitiert nach Quandt 2013: 153), die sich auf Wesentliches konzentrieren und Sinn stiften wollen. Narrative lassen sich daher als eine Übung verstehen, die „durch die bewusste Reduktion der beobachtbaren gesellschaftlichen Komplexität einzelne handhabbare Ausschnitte" (ebd.) bilden. Es liegt auf der Hand, dass Narrative als eine Erzählung, die Begeisterung erzeugen soll, notwendig zugespitzt und meistens hochselektiv sind (vgl. ebd.). Es kann also nicht darum gehen, alle möglichen Einwendungen und Entwicklungen zu berücksichtigen, wie es etwa bei Porter und Kramer in Bezug auf die CSR Entwicklung sehr deutlich wird, sondern vielmehr wahrgenommene Grundintuitionen aufzunehmen und auf dieser Basis eine einfache und affirmative Idee als neue Geschichte zu erzählen. Neue Narrative werden gebraucht, um ,academic, political, and practitioner discourse and thinking“ (Freeman et al. 2010: 269) zu orientieren. Freeman et al. (ebd.) zählen allein fünf verschiedene Narrative des Kapitalismus auf, die aber allesamt „retell the story of value creation and trade from the perspective of one stakeholder". Porters und Kramers Version des CSV ist dabei nichts anderes als - erstens - eine Wiedererzählung des investor capitalism, wie ihn bereits Friedman (2010) verteidigt hat (vgl. Freeman et al. 2010: 272), und - zweitens - eine grundsätzliche Verteidigung des Kapitalismus im Rahmen der Grundannahmen, die Freeman et al. (2010: 285) für alle Narrative des Kapitalismus identifiziert haben: „naive self-interest, the separation of business and morality, and the limited nature of valuable resources". 
Solange aber nur der Hammer zur Verfügung steht, sieht das Problem weiterhin aus wie ein Nagel (vgl. Donaldson 2014) und dann gilt das, was Streeck (2013: 256) in einem anderen Zusammenhang festgestellt hat: „Und solange die erstbeste Lösung keine ist, wird die zweitbeste zur erstbesten“. In genau diesem Sinne haben - bei aller Kritik - Hahn (2014), Dyllick (2014) sowie Lütge und von Liel (2014) Recht! Die damit einhergehende Gefahr ist, dass CSR als „side agenda“ auf Dauer gestellt und ökonomische Verantwortung als die gesellschaftliche Verantwortung des Unternehmens zementiert wird. Ernstzunehmende Legitimationsansätze, wie sie etwa in der Konstruktion der „Firm as Political Actor“ (vgl. Scherer et al. 2012; Scherer et al. 2014) vorliegen, rücken in den engen hermeneutischen Grenzen von Porter und Kramer und, wie wir vermuten dürfen, der business school community in weite Ferne. Vielleicht aber sehen wir ein neues Narrativ mit „Porter-Effekt“ dann, wenn sich die Forschung zum strategischen Management in Harvard wieder auf die umfassendere Konzeption zurück besinnt (vgl. Learned et al. 1967), die offensichtlich in den ausgehenden 1980er Jahren verloren gegangen ist.

\section{Literaturverzeichnis}

Albert, H. (1998): Marktsoziologie und Entscheidungslogik, Tübingen: Mohr.

Beschorner, T. (2013): Creating Shared Value: The One-Trick Pony Approach, in: Business Ethics Journal Review, Vol. 1/No. 17, 106-112, Link: https://bejreview.files.wordpress. com/2013/09/bejrv1n17beschorner.pdf (zuletzt abgerufen am 27.05.2015)

Crane, A. (2014): Premise of 'creating shared values' risks misleading MBA students, in: Financial Times, 20. April 2014. Link: http://www.ft.com/cms/s/2/7b19586c-add7-11e39ddc-00144feab7de.html\#axzz3UjSNyaA3 (zuletzt abgerufen am 02.03.2015).

Crane, A./Matten, D. (2010): Business Ethics, 3. Aufl., Oxford/ New York: Oxford University Press.

Crane, A./Palazzo, G./Spence, L. J./Matten, D. (2014a): Contesting the Value of "Creating Shared Value", in: California Management Review, Vol. 56/No. 2, 130-149.

Crane, A./Palazzo, G./Spence, L. J./Matten, D. (2014b): Reply, in: California Management Review, Vol. 56/No. 2, S. 151-153.

Donaldson, T. (2014): Shared Values that are lost in translation, Financial Times, 23. April 2014. Link: http://www.ft.com/cms/s/2/bfdfff94-b34c-11e3-b09d-00144feabdc0.html\# axzz3TXU9mili (zuletzt abgerufen am 02.03.2015).

Driver, M. (2012): An Interview With Michael Porter: Social Entrepreneurship and the Transformation of Capitalism, in: Academy of Management Learning \& Education, Vol. 11/No. 3, 421-431.

Drucker, P. (2002): Was ist Management? Das Beste aus 50 Jahren, München: ECON.

Drucker, P. (2002a/1974): Gesellschaftliche Auswirkungen und Probleme, in: Drucker, P. (2002): Was ist Management? Das Beste aus 50 Jahren, München: ECON, 73-93.

Drucker, P. (2002b/ 1974): Zwecke und Ziele eines Unternehmens, in: Drucker, P. (2002): Was ist Management? Das Beste aus 50 Jahren, München: ECON, 35-58.

Dyllick, T. (2014): The Opposing Perspectives on Creating Shared Values, in: Financial Times, 24. April 2014. Link: http://www.ft.com/intl/cms/s/2/88013970-b34d-11e3-b09d00144feabdc0.html\#axzz3TXU9mili (zuletzt abgerufen am 02.03.2015).

Dyllick, T. (1989): Management der Umweltbeziehungen. Öffentliche Auseinandersetzungen als Herausforderung, Wiesbaden: Gabler. 
Freeman, R. E. (1994): The Politics of Stakeholder Theory: Some Future Directions, in: Business Ethics Quarterly, Vol. 4/No. 4, 409-421.

Freeman, R. E./Harrison, J. S./Wicks, A. C./Parmar, B. L./Ce Colle, S. (2010): Stakeholder Theory. The State Of The Art, Cambridge: Cambridge University Press.

Friedman, M. (1970): The Social Responsibility of Business is to Increase its Profits, in: The New York Times Magazine, 13. September 1970. Link: http://www.umich.edu/ $\sim$ thecore/doc/Friedman.pdf (zuletzt abgerufen am 05.03.2015).

Friedman, M. (2010): Kapitalismus und Freiheit, 7. Aufl., München/ Zürich: Piper .

Grayson, D./Hodges, A. (2004): Corporate Social Opportunity! 7 Steps to Make Corporate Social Responsibility Work for Your Business, Sheffield: Greenleaf Publishing.

Hahn, T. (2014): Teaching CSV Will Not Breed Sustainable Business Leaders, in: Financial Times, 29. April 2014. Link: http://www.ft.com/intl/cms/s/2/0151e356-cee7-11e3ac8d-00144feabdc0.html\#axzz3UjSNyaA3 (zuletzt abgerufen am 02.03.2015).

Hamel, G. (2013): Worauf es jetzt ankommt. Erfolgreich in Zeiten kompromisslosen Wandels, brutalen Wettbewerbs und unaufhaltsamer Innovation, Weinheim: Wiley-VCH.

Hartman, L. P./Werhane, P. H. (2013): Proposition: Shared Value as an Incomplete Mental Model, in: Business Ethics Journal Review, Vol. 1/ No. 6, 36-43.

Heidbrink, L. (2007): Handeln in der Ungewissheit. Paradoxien der Verantwortung, Berlin: Kulturverlag KADMOS.

Hübscher, M. C. (2015): Creating Shared Value (CSV) - ein neues Konzept oder ein funktionales (ökonomistisches) Narrativ? Eine verstehende Rekonstruktion der Entwicklung des CSV, unveröffentlichtes Arbeitspapier.

Hübscher, M. C. (2011): Die Firma als Nexus von Rechtfertigungskontexten. Eine normative Untersuchung zur rekursiven Simultanität von Individuen und Institutionen in der Governanceethik, Marburg: Metropolis.

Kirsch, W./Seidl, D./Aaken, D. v. (2009): Unternehmensführung. Eine evolutionäre Perspektive, Stuttgart: Schäffer Poeschel.

Kramer, M. R. (2014): The MBA Blind Spot, in: Financial Times, 24. April 2014. Link: http://www.ft.com/intl/cms/s/2/145494e4-b34e-11e3-b09d-00144feabdc0.html\# axzz3d4yKlZ7p (zuletzt abgerufen am 02.03.2015).

Learned, E. P./Christensen, C. R./Andrews, K. R./Guth, W. D. (1967): Business Policy. Text and Cases, Homewood: Richard D. Irvin.

Lütge, C./v. Liel, B. (2014): Why CSV Makes Sense for Business Schools, in: Financial Times, 10. Juni 2014. Link: http://www.ft.com/intl/cms/s/2/f47575be-e280-11e3-a82900144feabdc0.html\#axzz3TXU9mili (zuletzt abgerufen am 02.03. 2015).

Porter, M. E. (2008): The Five Competitive Forces That Shape Strategy, in: Harvard Business Review, No. 86/Vol. 1. Link: http://www.exed.hbs.edu/assets/documents/hbrshape-strategy.pdf (zuletzt abgerufen am 03.03.2015).

Porter, M. E. (2003): Corporate Philanthropy. Taking The High Ground, Link: http://www.fsg.org/Portals/0/Uploads/Documents/PDF/Corporate_Philanthropy _Taking_the_High_Ground.pdf?cpgn=WP\%20DL $\% 20-\% 20$ Corporate $\% 20$ Philanthr opy $\% 20-\% 20$ Taking $\% 20$ the $\% 20$ High $\% 20$ Ground (zuletzt abgerufen am 04.03.2015).

Porter, M. E. (1999): Wettbewerbsstrategie. Methoden zur Analyse von Branchen und Konkurrenten, 10. Aufl., Frankfurt/ New York: Campus.

Porter, M. E. (1990): Nationale Wettbewerbskraft - woher kommt die? in: Harvard Business Manager, Jg. 12/Heft 4, 103-126.

Porter, M. E./Kramer, M. R. (2014): A Response to Andrew Crane et al's Article, in: California Management Review, Vol. 56/No. 2, 149-151. 
Porter, M. E./Kramer, M. R. (2012a): Die Neuerfindung des Kapitalismus, in: Harvard Business Manager, 3/2012, 14-29.

Porter, M. E./Kramer, M. R. (2012b): Shared Value: Die Brücke von Corporate Social Responsibility zu Corporate Strategy, in: Schneider, A./Schmidpeter, R. (Hrsg.): Corporate Social Responsibility, Berlin/Wiesbaden: Springer, 137-153.

Porter, M. E./Kramer, M. R. (2011): Creating Shared Value. How to Reinvent Capitalism - and Unleash a Wave of Innovation and Growth, in: Harvard Business Review, No. 89/Vol. 1-2, 62-77.

Porter, M./Kramer, M. R. (2007): Wohltaten mit System, in: Harvard Business Manager, 1/2007, 14-29.

Porter, M./Kramer, M. R. (2006): Strategy and Society: The Link between Competitive Advantage and Corporate Social Responsibility, in: Harvard Business Review, 84/12, 78-92.

Porter, M./Kramer, M. R. (2002): The Competitive Advantage of Corporate Philanthropy, in: Harvard Business Review, No. 80/Vol. 12. Link: http://www.fsg.org/tabid/191/ ArticleId/176/Default.aspx?srpush=true (zuletzt abgerufen am 02.03.2015).

Porter, M./Kramer, M. R. (1999): Philanthropy's New Agenda: Creating Value, in: Harvard Business Review, 121-130.

Prahald, C. K./Krishnan, M. S. (2009): Die Revolution der Innovation. Wertschöpfung durch neue Formen in der globalen Zusammenarbeit, München: Redline.

Priddat, B. P./Seele, P. (Hrsg.) (2008): Das Neue in Ökonomie und Management, Wiesbaden: Gabler.

Quandt, J. H. (2013): CSR zwischen Rhetorik und Nachhaltigkeit. Wie effektive Regulation gelingen kann, Marburg: Metropolis.

Scherer, A. G./Palazzo, G. (2012): The New Political Role of Business in a Globalized World A Review of a New Perspective on CSR and Its Implications for the Form, Governance, and Democracy, in: Corsten, H./Rothe, S. (Hrsg.): Nachhaltigkeit. Unternehmerisches Handeln in globaler Verantwortung, Wiesbaden, Gabler, 15-50.

Scherer, A. G./Palazzo, G./Matten, D. (2014): The Business Firm as a Political Actor: An New Theory of the Firm for a Globalized World, in: Business \& Society, Vol. 52/No. 2, 143-156.

Scholz, M./de los Reyes, G. (2015): Management von Shared Value - eine legitime Corporate Strategy, in: Schneider, A./Schmidpeter, R. (Hrsg.): Corporate Social Responsibility, 2. Aufl., Berlin/Wiesbaden: Springer, 543-556.

Schreck, P. (2012): Der Business Case for Corporate Social Responsibility, in: Schneider, A./Schmidpeter, R. (Hrsg.): Corporate Social Responsibility, 2. Aufl., Berlin/Wiesbaden: Springer, 67-87.

Sedlacek, T./Graeber, D. (2015): Revolution oder Evolution. Das Ende des Kapitalismus, München: Carl Hanser.

Streeck, W. (2015): Wie wird der Kapitalismus enden?, in: Blätter für deutsche und internationale Politik, 3/2015, 99-111.

Streeck, W. (2013): Gekaufte Zeit. Die vertagte Krise des demokratischen Kapitalismus, Berlin: Suhrkamp.

Ulrich, P. (2012): Gewinnprinzip oder prinzipiengeleitetes Gewinnstreben? Wirtschaftsethische Auflösung eines betriebswirtschaftlichen Tabus, in: Hahn, R./Janzen, H./Matten, D. (Hrsg.): Die gesellschaftliche Verantwortung des Unternehmens. Hintergründe, Schwerpunkte und Zukunftsperspektiven, Stuttgart: Schäffer Poeschel, 21-38.

Wieland, J. (2005): Normativität und Governance. Gesellschaftstheoretische und philosophische Reflexionen der Governanceethik, Marburg: Metropolis.

Wieland, J./Heck, A. (2013): Shared Value durch Stakeholder Governance, Marburg: Metropolis. 